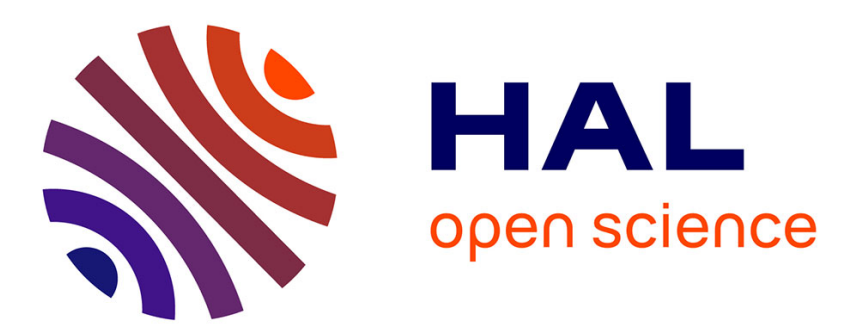

\title{
La perspective aérienne de Léonard de Vinci et ses origines dans l'optique d'Ibn al-Haytham (De aspectibus, III, 7) \\ Dominique Raynaud
}

\section{- To cite this version:}

Dominique Raynaud. La perspective aérienne de Léonard de Vinci et ses origines dans l'optique d'Ibn al-Haytham (De aspectibus, III, 7). Arabic Sciences and Philosophy, 2009, 19, pp.225-246. halshs00479826

\section{HAL Id: halshs-00479826 \\ https://shs.hal.science/halshs-00479826}

Submitted on 3 May 2010

HAL is a multi-disciplinary open access archive for the deposit and dissemination of scientific research documents, whether they are published or not. The documents may come from teaching and research institutions in France or abroad, or from public or private research centers.
L'archive ouverte pluridisciplinaire HAL, est destinée au dépôt et à la diffusion de documents scientifiques de niveau recherche, publiés ou non, émanant des établissements d'enseignement et de recherche français ou étrangers, des laboratoires publics ou privés. 


\title{
La perspective aérienne de Léonard de Vinci et ses origines dans l'optique d'Ibn al-Haytham (De aspectibus, III, 7)
}

\author{
Dominique Raynaud*
}

\begin{abstract}
The concept of aerial perspective has been used for the first time by Leonardo da Vinci (14521519). This article studies its dependence on Ptolemy's Optica and overall on the optical tradition inaugurated by Ibn al-Haytham's Kitâb al-Manâzir (d. after 1040). This treatise, that was accessible through several Latin and Italian manuscripts, and was the source of many Medieval commentaries, offers a general theory of visual perception emancipated from the case of the moon illusion, in which physical and psychological factors are closely combined. Atmospheric extinction (not refraction, which it is sometimes confused with) affects the conjectured size of remote objects. This phenomenon is also the core source for a pictorial rendering of depth, that is based onto a principle different from the diminution of size.

Résumé. Le concept de perspective aérienne a été introduit par Léonard de Vinci (1452-1519). L'article étudie sa dépendance vis-à-vis de l'Optique de Ptolémée et surtout de la tradition optique inaugurée par le Kitâb almanâzir d'Ibn al-Haytham (m. après 1040). Ce traité, accessible par plusieurs manuscrits latins et italien, qui a fait l'objet de nombreux commentaires médiévaux, offre une théorie générale de la perception visuelle émancipée du seul cas de l'illusion lunaire, dans laquelle les facteurs physiques et psychologiques sont étroitement associés. L'extinction atmosphérique (et non la réfraction, avec laquelle elle est parfois confondue) influence la perception de la taille des objets éloignés. Elle est aussi à l'origine d'une restitution picturale de la profondeur, fondée sur un principe autre que celui de la diminution des grandeurs.
\end{abstract}

\section{Origine du concept de perspective aérienne}

Léonard de Vinci (1452-1519) est tenu pour l'inventeur de la «perspective aérienne » (prospettiva aerea, prospettiva de’ perdimenti), à laquelle il consacre une vingtaine de passages, de trois à quatre-vingt-six lignes dans l'édition de Richter, parmi lesquels les suivants :

\footnotetext{
« È ci una altra prospectiva la quale chiamo aerea, imperoche per la varietà dell'aria si può [1] conosciere le diverse distantie di vari edifiti [...] Chiaro si uede essere [2] una aria grossa piu che l'altre, la quale confina colla terra piana, e quanto piv si leua in alto, piv e sottile e transparente ; le cose eleuate e grandi, che fieno da te lontane, la lor bassezza poco fia veduta perche la uedi per una linia che passa infra l'aria piv grossa e continvata [...] e per questa ragione, questa linia quanto piv s'allontana da te, di punto in punto sempre mvta qualità di sottile in sottile aria. [3] Adunque tu pittore, quando fai le montagnie, fa che di colle in colle sempre le bassezze sieno piv chiare che l'altezze, e quanto vi farai piv lontana l'una da l'altra fa le bassezze piu chiare, e quanto piv se leverà in alto, piv mostrera la verità della forma e colore » ${ }^{1}$.
}

\footnotetext{
* Université Pierre-Mendès-France (Grenoble) et CNRS UMR 8598 (Paris).

${ }^{1}$ Ashburnham I, fol. 10a, 17b (1492), The Notebooks of Leonardo da Vinci, ed. J. P. Richter (New York, 1970), vol. I, pp. 159, 160-162.
} 
Le terme de prospettiva aerea désigne trois faits interdépendants : [1] l'estimation des distances (optique physiologique et psychologique), [2] l'extinction atmosphérique dont elle procède (optique physique), [3] sa restitution picturale (savoir-faire artistique). Selon Vinci, cette extinction de la lumière est causée par les vapeurs humides (umidità vaporata), les vapeurs (vapori), la brume (nebbia), la fumée (fumo), les poussières (poluere) ou simplement une atmosphère dense (aria grossa, grossi umori). La perspective aérienne permet donc de conjecturer la distance d'un objet, qui apparaît d'autant plus estompé qu'il est éloigné. Il peut de ce fait exister des erreurs d'inférence : un objet dont la lumière est anormalement atténuée paraîtra anormalement éloigné et, par suite, plus grand (quoique ayant le même diamètre angulaire).

Vinci établit explicitement un rapport entre la perspective aérienne et la perception de la taille des astres à l'horizon. À la question « Perchè il sole pare magiore nel tramontare che di mezzo giorno che ci è presso ? », Vinci répond en invoquant tantôt la réfraction atmosphérique, tantôt la perspective aérienne :

[1] « Ogni chorpo che è visto per churvo mezo apparisce di magiore forma che non è » ${ }^{2}$.

[2] «E vidi l'aria sopra di me tenebrosa e'l sole che percotea la montagnia essere piu luminoso quiui assai che nelle basse pianure, perche minor grosseza d'aria s'interponea infra la cima d'esso monte e'l sole »'

En avançant cette deuxième explication, Léonard de Vinci apparaît comme un précurseur d'auteurs classiques - tels que Berkeley, Bouguer, Le Cat ou Euler ${ }^{4}$ - qui étudieront eux aussi la relation entre l'illusion lunaire et l'extinction atmosphérique.

Ces éléments (situation des astres à l'horizon, extinction vs. réfraction atmosphérique, perception de la distance, restitution picturale) ne sont pas autochtones en peinture. Ils appartiennent au corpus de l'optique, qui propose tout à la fois une théorie générale de la perception visuelle et une interprétation de l'illusion lunaire, ainsi nommée d'après son cas paradigmatique: « Pourquoi la Lune paraît-elle plus grande à l'horizon qu'en culmination ? ${ }^{5}$. Il faut alors retirer à Vinci la paternité du concept de perspective aérienne et se demander quelles sont ses sources. Cette question a été discutée par de nombreux savants de l'Antiquité et du Moyen Âge. Ptolémée écrit par exemple au Livre II de l'Optique:

\footnotetext{
${ }^{2}$ Ms. A, fol. 64a (1492), Notebooks, vol. II, p. 153.

${ }^{3}$ Ms. Leic., fol. 4a (ca. 1500-1510), Notebooks, vol. I, p. 162.

${ }^{4}$ G. Berkeley, «An Essay Towards a New Theory of Vision » (1709), in The Works of George Berkeley, Bishop of Cloyne (London, 1948): 143-239 ; P. Bouguer, Essay d'optique sur la gradation de la lumière (Paris, 1729) ; C. N. Le Cat, Traité des sens (Rouen, 1744); L. Euler, «Letters CX-CXIV » (1762), Letters of Euler to a German Princess, on Different Subjects in Physics and Philosophy (Bristol, 1997), II : 480-500.

${ }^{5}$ A. I. Sabra, «Psychology versus mathematics: Ptolemy and Alhazen on the moon illusion », E. Grant and J. E. Murdoch, eds., Mathematics and its Applications to Natural Philosophy in the Middle Ages. Essays in Honour of Marshall Clagett (Cambridge, 1987): 217-247.
} 
«Des objets situés en un même lieu, ceux qui sont plus brillants semblent plus proches. Dans ces sortes d'illusions, le critère pour voir [la distance de] l'objet estompé [rem occultam] n'est pas la longueur du rayon, \{quand la distance entre l'œil et l'objet est très grande\}, mais la différence [d'éclat] des couleurs. De la même façon, la position des objets lumineux [...] est estimée plus proche, tandis que les objets peu éclairés [habentes occultam claritatem] apparaissent plus éloignés quoique plus proches. C'est pourquoi, les peintres choisissent pour les objets qu'ils veulent montrer plus éloignés des couleurs nébuleuses et voilées [colores... aereos latentes] 》 (II, 124).

«Lorsque des objets sous-tendent des angles égaux à des distances égales, celui qui a la couleur la moins vive [minorem habet colorem] apparaît plus grand [...] L'objet dont la couleur est plus estompée [color est magis occultus] apparaît plus éloigné et du même coup est estimé plus grand » (II, 126).

«Se produit encore une illusion dans le raisonnement sur les distances et leur évaluation, comme il arrive quand nous regardons dans l'air où nous sommes. En effet, comme ce même air est plus dense [spissior] et plus coloré [magis coloratus] que les couches supérieures parce qu'il est le lieu d'une abondante évaporation qui monte de la terre et des eaux, il devient plus apte à capter la lumière [...] L'air qui apparaît le jour semble plus éloigné que tout, tandis que le Soleil et la Lune semblent plus proches à cause de leur éclat [propter claritatem]. [...] L'esprit pense que ce qui lui semble le plus reculé [res que nouissima ei uidetur] est plus grand que ce qui naturellement et réellement est plus éloigné et plus grand que le reste » (II, 139).

Ptolémée explique ces erreurs de jugement de taille, auxquelles se rattache l'illusion lunaire, par deux composantes. La première réside dans l'affaiblissement de la lumière : les objets ont moins d'éclat quand l'air est plus dense (spissior) et plus coloré (magis coloratus) à cause des vapeurs atmosphériques (exalatio). La deuxième composante réside dans la perception de la taille : les objets dont la luminosité est plus faible ou la couleur est plus pâle paraissent plus éloignés. Étant vus sous le même diamètre angulaire, ils paraissent plus grands.

\section{Héritage de la tradition alhazénienne}

Interrogeons-nous maintenant sur les conditions qui ont rendu possible le transfert de ce concept de l'optique à la perspective. Tout indique que ce transfert a été facilité par une œuvre issue - quoique émancipée - de la tradition ptoléméenne : l'optique d'Ibn al-Haytham (Alhazen, m. après 1040). Léonard de Vinci a pu connaître cette œuvre directement, dans ses versions latine $^{7}$ ou italienne ${ }^{8}$, ou indirectement, par le biais des commentaires médiévaux que cette œuvre a

\footnotetext{
${ }^{6}$ Optica, II, 124, 126, 139, A. Lejeune, L'Optique de Claude Ptolémée dans la version latine d'après l'arabe de l'émir Eugène de Sicile. Édition critique et exégétique, trad. française (Leiden, 1989), pp. 74-76, 83. Je remercie l'un des rapporteurs de m'avoir signalé que les mots cum multa fuerit distantia, restitués entre accolades $\{. .$.$\} ,$ n'avaient pas été traduits par Lejeune.

${ }^{7}$ A. M. Smith, Alhacen's Theory of Visual Perception. A Critical Edition, with English Translation and Commentary, of the First Three Books of Alhacen's De Aspectibus, 2 vols, Philadelphia, 2001 ; A. M. Smith, Al-
} 
suscités, en particulier ceux de Roger Bacon, Witelo, John Pecham ${ }^{9}$. Il existe des indices probants de l'affiliation de Vinci à cette tradition alhazénienne.

$1^{\circ}$ En atteste tout d'abord l'observation de A. Mark Smith selon laquelle Léonard a substitué au rayon visuel euclido-ptoléméen le rayon lumineux alhazénien, «si bien que son analyse de la vision est intégralement basée sur le cône de radiation décrit par Alhazen et ses commentateurs $»^{10}$.

$2^{\circ}$ D'autres éléments sont fournis par Graziella Federici Vescovini, qui a non seulement établi que les Commentarii de Lorenzo Ghiberti dépendaient du De li aspetti - traduction italienne du De aspectibus datant de la fin du XIV siècle - mais qu'il était fondé de penser que ce texte a circulé largement dans les milieux artistiques du Quattrocento ${ }^{11}$.

$3^{\circ}$ En attestent enfin plusieurs parallèles textuels, dont l'étude systématique ne relève pas du cadre de cet article $^{12}$. Un de ces parallèles concerne les effets de réflexion colorée :

Vinci : « La superfitie d'ogni corpo oppacho participa del colore del suo obbietto [...] E tanto più si tigne la superfitie de corpi opachi del colore del suo obietto quanto tal superfitie è più biancha* e'l colore dello obietto più luminoso o aluminato ${ }^{\star}$ (Ms. W, fol. 240b, Notebooks, II, p. 144).

Alhazen : «Et si positum fuerit prope illud corpus corpus album* clare albedinis* et fuerit illud corpus in umbra et in luce debili, apparebit super ipsum color corporis, sicut narravimus superius. Deinde si appropinquet illud corpus album donec sit in lumine solis, latebit ille color qui est super eum, et si revertatur ad umbram, apparebit ille color fulgens ${ }^{\circ}$ super ipsum » (Op., I, 4.14, Smith, Alhacen's, I, p. 7).

hacen's on the Principles of Reflection. A Critical Edition, with English Translation and Commentay, of Books 4 and 5 of Alhacen's De Aspectibus, 2 vols, Philadelphia, 2006. Livres 6 et 7 (réfraction) en préparation.

${ }^{8}$ G. Federici Vescovini, « Contributo per la storia della fortuna di Alhazen in Italia: Il volgarizzamento del ms. vat. 4595 e il 'Commentario Terzo' del Ghiberti », Rinascimento, 5 (1965): 17-49; « La fortune du 'De aspectibus' de Alhazen (Kitâb al-Manâzir) au moyen âge latin », Archives internationales d'histoire des sciences, 40 (1990): 220-238 ; «Alhazen vulgarisé: Le De li aspecti d'un manuscrit du Vatican (moitié du XIVe siècle) et le troisième Commentaire sur l'optique de Lorenzo Ghiberti », Arabic Sciences and Philosophy, 8 (1998): 67-96.

${ }^{9}$ Vinci cite explicitement les œuvres de Bacon : rugero bachone (Br. Museum, fol. 71v), de Witelo : in uitolone he 805 chonclusioni in prospettiua (B. fol. 58r), vitolone in san marco (Br. Museum, fol. 79v), de Pecham, dont Vinci reproduit mot-à-mot certains passages, par exemple: La luce operando... (Cod. Atl., 594a, Notebooks, I, p ; 24) ; Lucem operari... (Perspectiva communis, I,1, D. C. Lindberg, John Pecham and the Science of Optics (Madison, 1970), p. 62. Ces trois auteurs ont tous étudié l'illusion lunaire: Bacon, Perspectiva, II, III, 6, Roger Bacon and the Origins of "Perspectiva" in the Middle Ages, ed. D. C. Lindberg (Oxford, 1996), pp. 226-228 ; John Pecham, Tractatus de perspectiva, XII, ed. D. C. Lindberg (St. Bonaventure, 1972), pp. 67-68.

${ }^{10}$ Smith, Alhacen's, I, p. cli, n. 340.

${ }^{11}$ «Como abbiamo cercato di dimostrare in altra occasione, questo testo fu largamente utilizzato dal Ghiberti nel suo Commentario Terzo [...] non vediamo il motivo di escludere che Brunelleschi potesse esserne venuto a conoscenza [...] un volgarizzamento in italiano ne doveva circolare a Firenze agli inizi del Quattrocento », G. Federici Vescovini, « La prospettiva del Brunelleschi. Alhazen e Biagio Pelacani a Firenze », Filippo Brunelleschi. La sua opera e il suo tempo (Firenze, 1980): 333-348, p. 342-343.

${ }^{12}$ Nous laissons de côté le cas de la camera obscura (Notebooks, I, pp. 44-45) qui est issue de la même tradition. Ce dispositif (al-bayt al-muzzlim) est exposé dans le traité Sur la forme de l'éclipse (Maqâla fi sûrat al-kusûf), E. Wiedemann, «Über die camera obscura bei Ibn al-Haitam », Sitzungsberichte der physikalisch-medizinischen Sozietät in Erlangen, 46 (1914): 155-169, dans les trois premiers chapitres, non traduits en latin, du Kitâb almanâzir (A. I. Sabra, The Optics of Ibn al-Haytham (London, 1989), I, pp. 13-15), mais aussi en divers passages de la version latine du De Aspectibus (cf. par exemple Smith, Alhacen's, I, p. 121, 132). 
Les deux passages renferment la même idée, quoiqu'elle y soit exprimée sous une forme un peu différente. Les corps réfléchissent la couleur des objets avoisinants et cette réflexion colorée est d'autant plus forte que le corps est plus blanc (corpus album clare albedinis ; superfitie più biancha) et que la couleur est plus lumineuse (colore aluminato ; color fulgens). L'étude des sources pose deux problèmes spécifiques.

Le premier problème est que Vinci - par esprit d'indépendance ou compréhension limitée de l'optique - insère des exemples classiques dans des développements nouveaux qui peuvent aller jusqu'à l'inversion. La perception de la transparence en offre un exemple :

Vinci: «Il mezzo ch'è infra l'ochio e la cosa vista trasmuta essa cosa in nel suo colore, come il vetro rosso* fa che ciò che l'ochio vede dopo lui pare rosso » (Tr. fol. 75, Notebooks, I, p. 160).

Alhazen: "Cum cristallo continuatur corpus rubeum*, alia cristalli facie visui opposita, existimabit videns colorem cristalli esse rubedinem » (III, 6.16, Smith, Alhacen's, I, p. 299).

Alors qu'Alhazen prend l'exemple du verre coloré pour illustrer le fait que le milieu transparent prend la couleur du corps rouge placé derrière lui ; Vinci en fait une illustration du fait que le corps prend la couleur du verre coloré placé devant lui.

Le second problème inhérent à l'étude des parallèles est celui de la forte interdépendance des optiques issues de la tradition alhazénienne. Le phénomène d'irisation en offre un exemple :

Vinci : «Molti sono li ucielli nelle varie region del mondo, nelle penne de quali si vede bellissimi colori gienerarsi nelli lor diuersi movimenti, come far si vede infra noi alle penne delli paghoni* o nelli colli delle cholonbe ${ }^{\circ} »$ (Windsor F., fol. 145 Ab, Notebooks, II, p. 153).

Alhazen : «Et iterum invenimus pennas pauonis* et pannum qui dicitur amilialmon quod diversatur in colore apud visum in diversis temporibus diei secundum diversitatem lucis orientis super ipsa » (Op., I, 4.25, Smith, Alhacen's, I, p. 10).

Bacon : «Et iterum non uidemus, quod secundum diuersitatem casus lucis ejusdem super eandem rem mutatur aspectus, et apparet uisui color diuersus, ut in collo columbae ${ }^{\circ}$ secundum quod uertit collum diuersis sitibus ad lucem et sic de cauda pauonis*» (Perspectiva, I, 5, Bridges, II, 32).

Pecham : «Amplius hoc idem apparet in collo columbae ${ }^{\circ}$, quod cum unius sit coloris, variis tamen aspectibus varie illustratum sub differenti specie oculo presentatur » (Perspectiva communis, I, 12, Lindberg, p. 86).

Witelo : «Visus ergo non recipit formam coloris rei visae, nisi ex luce admixta cum forma coloris, et propter hoc alterantur colores multarum rerum apud visum per alterationem lucis orientis super ipsas » (Perspectiva, III, 1, Risner, p. 85).

Si l'on compare ces textes, on observe que l'optique de Witelo est la seule à ne pas offrir de concordance exacte. Vinci a pu trouver colli delle cholombe chez Bacon ou Pecham; penne delli paghoni dans l'optique d'Alhazen directement - à moins de supposer qu'il ait altéré les mots cauda pauonis de Bacon. Les textes de la tradition alhazénienne sont dans des rapports de proximité tels qu'il est parfois difficile d'identifier les sources utilisées.

L'indépendance de Vinci et la proximité des sources compliquent donc la recherche des textes qui ont modelé le concept de perspective aérienne. Seule une étude systématique permettrait de savoir si Vinci a utilisé l'optique d'Alhazen ou celle de ses commentateurs; la version 
latine plutôt que la version italienne. Une telle comparaison dépasse les objectifs que nous nous sommes assignés dans cet article.

Toutefois, la parenté avérée entre toutes les versions nous autorise à étudier l'origine plutôt que la provenance du concept de perspective aérienne. Nous pouvons nous référer à l'optique d'Ibn al-Haytham, non pas en tant que source unique des réflexions de Vinci, mais en tant que matrice des versions et des commentaires qui en sont issus. L'avantage de ce procédé est qu'il permet de saisir la pensée dans son unité, dans un texte qui n'a pas subi de corruption, de coupe ou de réinterprétation majeure. Dans la suite du texte, «origine » signifiera donc inscription dans la tradition alhazénienne, plutôt que dépendance étroite à l'égard de telle ou telle version latine ou italienne du Kitâb al-manâzir .

Mais par ailleurs, pourquoi ne pas prendre comme point de départ de cette enquête le texte de Ptolémée ? Les historiens de l'optique s'accordent à penser que les contributions de Ptolémée et d'Alhazen sont proches relativement à la question de la perspective aérienne ${ }^{13}$. Doit-on alors considérer que l'optique d'Ibn al-Haytham aurait influencé sa descendance davantage par son accessibilité matérielle que par son contenu ? Avec vingt-quatre manuscrits, dontune version italienne,${ }^{14}$ contre quinze ${ }^{15}$, le De aspectibus d'Alhazen fut incontestablement un texte plus diffusé que l'optique de Ptolémée. Toutefois, certains éléments nous portent à penser que le contenu propre du De aspectibus a également joué un rôle dans la définition de la perspective aérienne. L'optique d'Ibn al-Haytham, fondée sur le paradigme de l'intromission des rayons lumineux - contrairement à la théorie ptoléméenne du rayon visuel - était à même de faciliter l'application des concepts de l'optique à la perspective : $1^{\circ}$ elle contenait de nombreux passages sur le jugement esthétique et la perception de la beauté ( $h u s n / p u c h r i t u d o$ ) et de la laideur $(q u b \underline{h} / \text { turpitudo })^{16} ; 2^{\circ}$ elle abordait la question de la ressemblance et du rendules textures dans les peintures (nuqûsh/picturae) $)^{17} ; 3^{\circ}$ elle proposait une théorie générale des erreurs de perception de la taille, englobant celle envisagée par Ptolémée ; $4^{\circ}$ elle introduisait, enfin, une explication combinant étroitement les facteurs physiques et psychologiques, laquelle, comme nous le verrons, ne pouvait que faciliter l'acclimatation des concepts de l'optique en perspective. Nous étudierons ici les principaux éléments de la théorie de la perception visuelle d'Ibn al-Haytham qui servirent de matrice au concept de « perspective aérienne ».

\footnotetext{
${ }^{13}$ Smith, Alhacen's, II, p. 638, n. 67.

${ }^{14}$ Deux nouveaux manuscrits se sont ajoutés à la liste de Lindberg, portant le total à vingt-quatre manuscrits, D.

C. Lindberg, A Catalogue of Medieval and Renaissance Optical Manuscripts (Toronto, 1975), pp. 17-19; Smith, Alhacen's, I, pp. clv-clxi.

${ }^{15}$ Lindberg, Catalogue, pp. 74-75.

${ }^{16}$ Kitâb al-manâzir , I, 3 et III, 7; Sabra, Optics, I, pp. 200-206, 300-311, 313-314, 322-323, 330-331, 338-339, 344-345, 355-356, 363-364.

${ }^{17}$ Ibid., III, 7.39-43, 85-86 ; Sabra, Optics, I, pp. 295-297, 309-310 ; Smith, Alhacen's, I, pp. 311, 317.
} 


\section{3. Évolution des conceptions d'Ibn al-Haytham}

Selon Abdelhamid I. Sabra ${ }^{18}$, la théorie d'Ibn al-Haytham serait marquée par l'abandon d'une explication optico-géométrique de l'illusion lunaire au profit d'une explication psychologique. La question est de savoir comment Ibn al-Haytham a basculé de l'une à l'autre de ces positions ${ }^{19}$. En examinant l'ordre dans lequel apparaissent les traités d'Ibn al-Haytham dans le ms. de Lahore (556 H./1161) et dans les catalogues d'Ibn al-Qifțî́ ${ }^{20}$ et Ibn Abî Usaybi 'a ${ }^{21}$, et en tenant compte des références internes qu'on trouve dans les traités, Sabra a proposé la chronologie suivante :

1. Commentaire de l'Almageste [Sharh al-Majistî̂], av. le 10 février 1027 ;

2. Optique [Kitâb al-manâżir];

3. Solution des doutes relatifs à l'Almageste formulés par un savant (Ibn Ma'dân) [ [Hall shukûk fî kitâb alMajistî yushakkiku fîhâ ba 'du ahli al-'ilm] ;

4. Épître sur la visibilité des astres [Risâla fî ru'yat al-kawâkib];

5. Doutes sur Ptolémée [Maqâla fỉ al-shukûk'alâ Batlamiyûs]

Sabra se réfère aux bibliographes Ibn Abî Usaybi‘a et al-Qiftî pour attribuer à Ibn alHaytham deux traités (désormais perdus) : Livre dans lequel est résumée la science de l'optique selon les livres d'Euclide et de Ptolémée, Traité d'optique selon la méthode de Ptolémée. Il en tire la conséquence qu'Ibn al-Haytham serait passé - en même temps qu'il aurait découvert l'Optique de Ptolémée - d'une explication optico-géométrique de l'illusion lunaire (par réfraction) à une explication psychologique. La dichotomie « substance/accident» lui aurait permis de minimiser son erreur de jeunesse sans se désavouer. La réfraction, devenue « cause accidentelle », fait place à la théorie du ciel aplati, désormais promue au rang de « cause permanente ».

\footnotetext{
${ }^{18}$ Sabra, «Psychology versus mathematics »; Sabra, Optics, passim; A. I. Sabra, A. Heinen, « On seeing the stars I, II », Zeitschrift für Geschichte der arabisch-islamischen Wissenschaften, 7 (1991/2): 31-72 et 10 (1995/6): 1-59 ; al-Shukûk 'ala Bațlamyûs, edited by A. I. Sabra and N. Shehaby (Le Caire, 1971), dont la partie sur l'optique a été publiée séparément: A. I. Sabra, «Ibn al-Haytham's criticisms of Ptolemy's Optics », Journal of the History of Philosophy, 4 (1966): 145-149.

${ }^{19}$ Nous laissons de côté la théorie d'Aristote, Meteorologica, III, 4, 373b, qui explique l'illusion lunaire par la réflexion. Hormis les contributions classiques de Strabon, Olympiodore, Ptolémée et Théon, signalons celles de

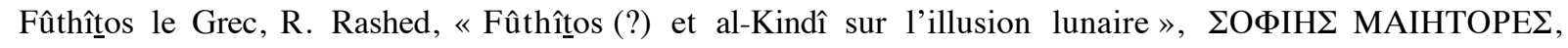
"Chercheurs de sagesse". Hommage à Jean Pépin, éd. M.-O. Goulet-Cazé et al. (Paris, 1992), pp. 533-559; alKindî (m. ca. 873), Les Corps immergés dans l'eau [al-Ajrâm al-ghâ'i isa fì al-mâ'], éd. R. Rashed, L'Optique et la catoptrique (Leiden, 1997), pp. 424-427; Ahmad Ibn 'Îsâ (Xe siècle), al-Manâzir wa al-marâyâ al-muhriqa, éd. R. Rashed, L'Optique et la Catoptrique, p. 127 ; Abû Hâsim al-Jubbâ'î (m. 933), qui explique aussi l'atténuation de la lumière solaire à l'horizon par la vapeur d'eau et la poussière, cf. Sabra, Optics, II, pp. 109110.

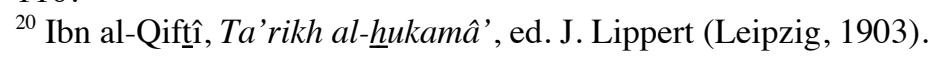

${ }^{21}$ Ibn Abî Unsaybi‘a, 'Uyûn al-anbâ' fî tabaqât al-atibbbâ', ed. A. Müller (Könisberg, 1884), ed. N. Ridâa (Beyrouth, 1956).
} 
La faiblesse de cette thèse d'une conversion intellectuelle est qu'elle s'appuie sur des données conjecturales. Les arguments avancés par Sabra pour identifier le mathématicien Abû 'Alî

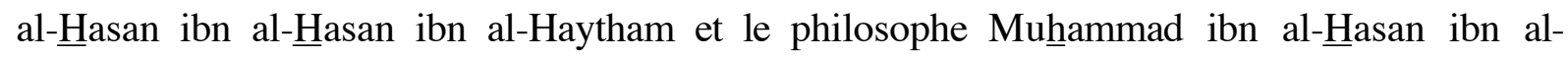
Haytham $^{22}$, souvent confondus par les anciens bibliographes, ont été remis en question par Roshdi Rashed ${ }^{23}$. La distinction qu'il propose est créditée par un élément en relation directe avec le sujet de cet article : alors que Muhammad lephilosophe explique l'illusion lunaire par la réflexion (comme Aristote et al-Kindî), l'auteur de l'Optique, Abû 'Alî al-Ḧasan, ne mentionne jamais cette explication. Si l'on admet l'existence de deux personnages distincts, le Commentaire devrait être exclu du «Tableau récapitulatif des oeuvres d'Ibn al-Haytham [le mathématicien] $\gg^{24}$. Il est par ailleurs douteux que l'Épître puisse être postérieure à l'Optique, car elle utilise systématiquement la théorie émissionniste du rayon visuel [shu ' $\hat{a}$ ' al-bas ar] supplantée par l'intromissionisme dans l'Optique. Cela permet de reclasser les traités consacrés à la perspective aérienne ou à l'illusion lunaire :

1. [no. 78] Épître sur la visibilité des astres [Risâla fî̀ ru'yat al-kawâkib], tentative pour accorder l'Optique et l'Almageste de Ptolémée sur le grandissement des astres à l'horizon. Il existe des parallèles textuels entre l'Épître... et la Solution des doutes... Ibn al-Haytham utilise le concept de rayon visuel [shu'âa' al-basar] disparu de l'Optique. Il recourt à la réfraction accidentelle et signale l'Optique en chantier : «Dans ce traité, je veux être bref »; «De cela, nous reparlerons dans un autre traité ${ }^{25}$;

2. [no. 57] Optique [Kitâb al-manâżirir], cf. principalement Livres II, III, VII ;

3. [no. 20] Solution des doutes relatifs à l'Almageste formulés par un savant (Ibn Ma'dân) [ㅂall shukûk fî̀ kitâb al-Majistî yushakkiku fihhâ ba'du ahli al-'ilm]. Ibn al-Haytham ne cite pas l'Épittre. Il mentionne la théorie de la réfraction accidentelle et reprend la distinction entre «cause accidentelle » et «cause permanente » à son Optique, qui est citée en I, 6 et III, 6 : «J'ai montré dans mon livre d'Optique »; «J'ai expliqué ce point dans mon livre d'Optique $»^{26}$;

4. [no. 85] Doutes sur Ptolémée [Maqâla fî al-shukûk 'alâ Batlamiyûs]. En dépit des parallèles textuels qui lient l'Épître et les Doutes, ces derniers sont postérieurs à l'Optique qui est citée : «J'ai montré dans la seconde maqâla de mon livre d'Optique ${ }^{27}$. Il ne mentionne plus la théorie psychologique de l'illusion lunaire. Accord avec Sabra qui donne les Doutes postérieurs à l'Optique et « rédigés probablement après la correspondance avec Ibn Ma'dan $»^{28}$.

\footnotetext{
${ }^{22}$ A. I. Sabra, « One Ibn al-Haytham or Two? An exercise in reading the bio-bibliographical sources », Zeitschrift für Geschichte der Arabisch-Islamischen Wissenschaften, 12 (1998): 1-50.

${ }^{23}$ R. Rashed, «Fûthîtos (?) et al-Kindî̀, p. 543, n. 25.

${ }^{24} \mathrm{R}$. Rashed, Les Mathématiques infinitésimales du IXe au XIe siècle, vol. II: Ibn al-Haytham (London, 1993), pp. 512-534.

${ }^{25}$ Sabra et Heinen, «On seeing the stars », pp. 55, 67.

${ }^{26}$ Sabra, « On seeing the stars, II », pp. 35, 46.

${ }^{27}$ Sabra, «Ibn al-Haytham's criticisms of Ptolemy's Optics », p. 146.

${ }^{28}$ Sabra, «Ibn al-Haytham's criticisms », p. 145; Sabra, Optics, II, p. xxxix ; Sabra, « On seeing the stars, II », p. 58.
} 
Les oeuvres qui reviennent en propre à Ibn al-Haytham le mathématicien attestent que : $1^{\circ}$ l'Optique sert de trame à tous ces écrits : elle est en chantier au moment où l'Épître est rédigée, et elle est citée dans tous les autres textes traitant de l'illusion lunaire ; $2^{\circ}$ l'explication par les causes « accidentelle» (vapeurs réfractives) et «permanente » (théorie du ciel applati) ne marque pas le parachèvement des recherches d'Ibn al-Haytham sur les erreurs de la vision : elle disparaît en effet du dernier traité.

\section{Les explications de l'illusion lunaire du Livre VII}

C'est à la toute fin du Livre VII, qui est intégralement consacré à l'étude de la réfraction, qu'Ibn al-Haytham introduit une discussion de l'illusion lunaire. Il avance deux explications, l'une présentée comme « cause permanente », l'autre comme « cause accidentelle ».

\subsection{Le ciel aplati : cause permanente}

Rappelons le texte d'Ibn al-Haytham (cf. Figure 1) :

«La vue n'éprouve pas la concavité de la surface du ciel, qu'elle perçoit plane, et croit les astres disposés sur cette surface. Elle perçoit par conséquent des astres distincts et égaux comme ayant des tailles inégales, parce qu'elle rapporte à une grande distance l'angle au centre de l'oeil sous-tendu par l'astre proche de l'horizon, alors qu'elle rapporte à une petite distance l'angle sous-tendu par l'astre situé au milieu du ciel. Ainsi, elle percevra l'astre proche de l'horizon plus grand que l'astre [situé] au milieu du ciel ou proche du zénith. [...] C'est là une erreur de la vue constante et permanente parce que sa cause est constante et permanente $»^{29}$.

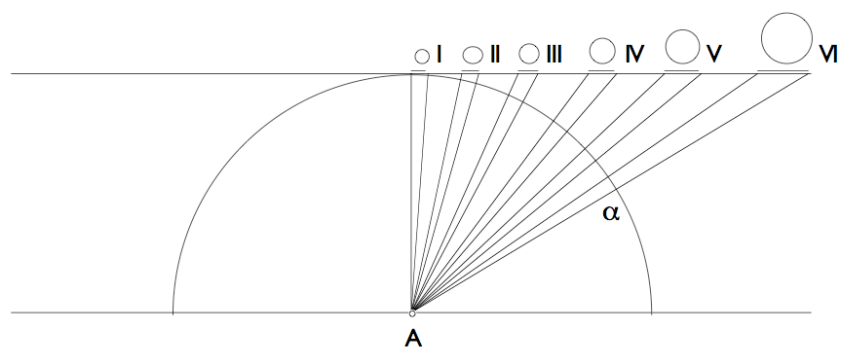

Dans l'hypothèse du ciel horizontal, l'observateur étant en $\mathrm{A}$, la Lune ou le Soleil ont un diamètre angulaire constant $\alpha$ qui découpe des segments inégaux $\mathrm{I}<$ II $<\ldots<$ VI.

\section{Figure 1. Théorie du ciel aplati}

Telle qu'elle se présente dans l'Optique, la « cause permanente » est due au fait que la couleur du ciel est uniforme. Comme les objets qui ont des couleurs uniformes sont généralement des surfaces planes, nous croyons le ciel horizontal. Alors des angles égaux (i.e. le diamè-

\footnotetext{
${ }^{29}$ Kitâb al-manâzinir, VII, 7.55 ; Sabra, « Psychology versus mathematics », pp. 238-241 ; Opticae thesaurus, éd. Risner, p. 281.
} 
tre angulaire de la Lune ou du Soleil) découpent des segments inégaux d'autant plus grands qu'il sont loins du zénith : le même astre est perçu plus grand à l'horizon.

\subsection{Les vapeurs réfractives : cause accidentelle}

\section{Voici le texte d'Ibn al-Haytham (cf. Figures 2 et 3):}

«L'agrandissement des objets célestes à l'horizon peut avoir une autre cause. Cette cause intervient si une vapeur épaisse est interposée entre l'oeil et l'astre positionné sur l'horizon, si la vapeur est au voisinage de l'horizon et ne s'élève pas vers le milieu du ciel, mais forme plutôt la section d'une sphère dont le centre est le centre du monde parce qu'elle entoure la terre. Si une telle section se termine avant le milieu du ciel, alors la surface [de la vapeur] qui fait face à l'oeil est plane. [...] Par conséquent, la vue percevra la forme [de l'astre] exactement comme elle percevrait des objets placés dans la vapeur, à savoir que la forme traversera la vapeur en ligne droite puis sera réfractée à la surface de la vapeur faisant face à l'oeil, cette réfraction étant hors de la normale à la surface de la vapeur (qui est une surface plane), puisque l'air proche de l'oeil est plus rare que l'épaisse vapeur. Il s'ensuit que la forme apparaîtra plus large qu'elle ne serait, si elle était vue [au moyen de rayons] rectilignes. [...] C'est là une cause accidentelle qui intervient toujours dans certaines régions de la terre, et occasionnellement en d'autres, mais qui n'est pas permanente $»^{30}$.

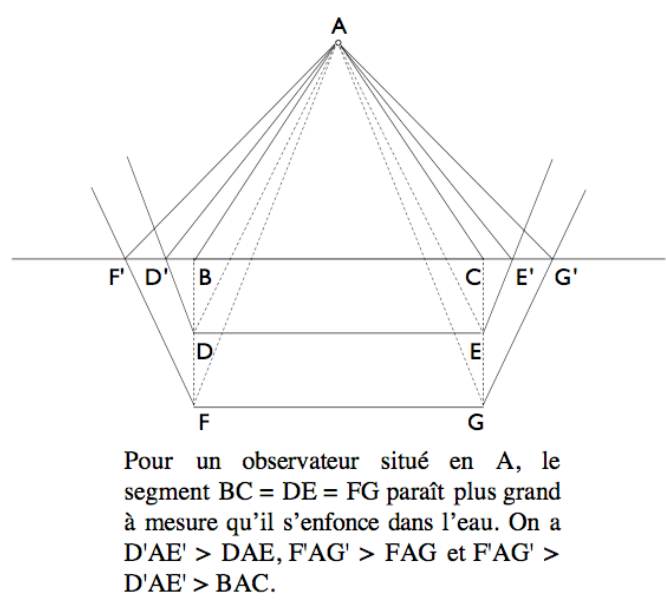

Figure 2

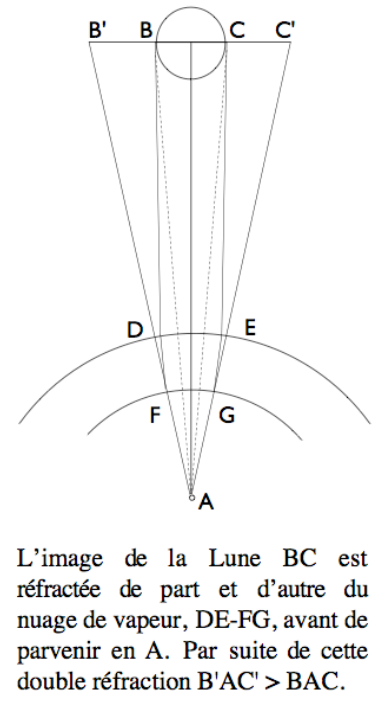

Figure 3

${ }^{30}$ Ibid., pp. 242-243. La Solution des doutes de l'Almageste, II.6-III.1, présente un début de reconnaissance de la réfraction dans les milieux inhomogènes : «Lorsque les astres se trouvent derrière la vapeur épaisse de l'horizon, ils seront vus plus grands en raison de la densité de vapeur, même si la vapeur ne présente aucune surface définie sur laquelle se produit la réfraction [...] La vapeur n'offre pas de surface que le rayon visuel puisse rencontrer, pas plus que le rayon ne passe successivement dans des milieux de densités différentes, mais plutôt traverse une seule et unique vapeur jusqu'à ce qu'il en sorte », Sabra, « On seeing the stars, II », pp. 43-45. Ibn al-Haytham dit que la vapeur ne présente aucune surface de séparation définie et que le rayon ne passe pas d'un milieu réfrangible à un autre, comme dans le cas classique. Il suppose une variation continue d'indice - autrement dit un gradient d'indice dirigé vers le centre du nuage typique des milieux inhomogènes. Mais nous sommes encore loin de $\mathrm{P}$. Bouguer, «Sur les réfractions astronomiques dans la zone torride », Histoire de l'Académie Royale des Sciences (1739): 45-48. 
L'explication, qui ne diffère de celle de Ptolémée que par son statut de « cause accidentelle ${ }^{31}$, est fondée sur le pouvoir réfractif supposé des vapeurs humides à l'horizon. - Ibn alHaytham postule : $1^{\circ}$ que ces vapeurs sont confinées dans la région de l'horizon ; $2^{\circ}$ qu'elles constituent une lentille plan-convexe, qui présente une calotte sphérique extérieure de même centre que la terre, et une face plane tournée vers l'oeil ; $3^{\circ}$ que la vapeur est plus dense que l'air. Dans ces conditions, il y aurait réfraction des rayons lumineux sur la lentille de vapeur, ce qui produirait un grandissement de l'image des $\operatorname{astres}^{32}$. Cette conclusion résulte de deux confusions : $1^{\circ}$ une confusion de nature entre réfringence et densité de vapeur; $2^{\circ}$ une confusion d'échelle : la sphère terrestre est si grande que sa section par un petit diamètre angulaire est assimilable à un plan sur lequel les rayons ne sont pas réfractés.

\section{La théorie de la perception visuelle du Livre II}

Le schématisme de la thèse selon laquelle l'illusion lunaire aurait été expliquée d'abord par l'optique géométrique, ensuite par la psychologie de la vision, doit également être nuancé. On trouve en fait dans l'oeuvre d'Ibn al-Haytham, non pas deux théories de l'illusion lunaire, mais de multiples explications - qui procèdent du cadre général de sathéorie de la perception visuelle (Livre II).

La perception visuelle s'appuie tantôt sur la sensation (ihhsâs/sensatio), tantôt sur la reconnaissance (ma'rifa/cognitio), tantôt sur l'inférence (istidlâl/argumentatio), tantôt sur une combinaison des trois. Les termes sont assez labiles pour qualifier le cas de l'inférence : Ibn alHaytham parle de jugement (al-tamyîz/distinctio), de comparaison (qiyâs/comparatio) ou de raisonnement (istidlâl-qiyâs/ratio-ratiocinatio) ${ }^{33}$. L'inférence de taille est tantôt passible d'une

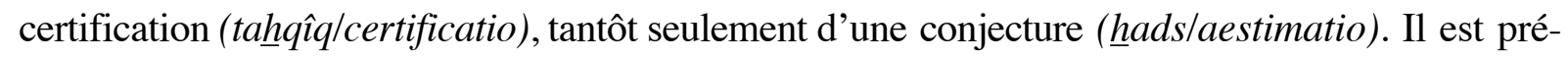
cisé que la capacité d'inférer la taille des objets est acquise par l'habitude (al-'ada, al-ma'lûf) et constitue une faculté inconsciente ou, plus précisément, non intentionnelle.

Partons du fait que l'illusion lunaire est un problème de jugement de taille. La taille est perçue par inférence à partir du diamètre angulaire, de la position et de l'évaluation de la distance ${ }^{34}$. Mais le diamètre angulaire lunaire variant peu et la position n'étant pas en jeu, l'illusion résulte d'une erreur d'appréciation des distances. La « grandeur de la distance » (kammiya/miqdâr) -

\footnotetext{
${ }^{31}$ Ibn al-Haytham se démarque trop lorsqu'il écrit que « [pour Ptolémée] le soleil est vu plus grand à l'horizon que lorsqu'il est au milieu du ciel seulement parce qu'une vapeur humide entourant la terre s'interpose entre lui et l'oeil », al-Shukûk 'alâ Bațlamyûs, éd. Sabra, « Ibn al-Haytham's criticism », p. 148 (mes italiques). En réalité, cette explication n'a rien d'exclusif chez Ptolémée.

${ }^{32}$ C'est l'une des explications retenues par Roger Bacon (Perspectiva, III, II, 4, éd. Lindberg, pp. 312-316) et par John Pecham (Tractatus de perspectiva, XII, éd. Lindberg, pp. 67-68 ; Perspectiva communis, I, 82 et III, 12 , éd. Lindberg, pp. 152, 222).

${ }^{33}$ Kitâb al-manâzirir, II, 3.150, 154 ; Sabra, Optics, I, pp. 179, 181 ; Smith, Alhacen's, I, pp. 172-173, 176.

${ }^{34}$ Ibid., II, 3.135 ; Sabra, Optics, I, pp. 173-174 ; Smith, Alhacen's, I, pp. 164 sq.
} 
distincte de la «distance » $\left(b u^{\prime} d\right)$ - est soit certifiée, soit conjecturée $e^{35}$. Dans le cas de la Lune, cette distance ne peut être que conjecturée. Il convient donc de reprendre l'examen des facteurs qui peuvent affecter cette conjecture.

\section{La théorie de la perspective aérienne du Livre III}

La théorie liant l'extinction atmosphérique aux erreurs de perception est exposée dans le texte fondamental de l'Optique et citée dans les réponses à Ibn Ma'dân. Le plan du Livre III est proprement combinatoire, puisque l'examen des erreurs visuelles est conditionné par les distinctions et catégories exposées antérieurement :

$1^{\circ}$ Les propriété visibles sont constituées de deux «sensibles propres »: lumière, couleur, et de vingt « sensibles communs »: distance, position, volumétrie, forme, taille, séparation et continuité, nombre, mouvement et repos, texture et planéité, transparence et opacité, ombre et obscurité, beauté et laideur, similarité et différence (II, 3.44) ;

$2^{\circ}$ La perception s'opère par sensation pure, reconnaissance ou inférence (III, 4.1) ;

$3^{\circ}$ Les conditions normales de vision sont au nombre de huit, à savoir : distance, position, lumière, taille et opacité du visible, transparence du milieu, durée de vision, normalité de l'oeil (III, 3.1).

Les erreurs visuelles naissant de conditions anormales de vision, celles-ci doivent être croisées avec les modalités de perception et les propriétés visibles. Mais, les sensibles propres étant perçus exclusivement par sensation (III, 5), et la reconnaissance étant un jugement essentiellement fondé sur la similarité et la différence (III, 6), Ibn al-Haytham ne fait jouer pleinement la combinatoire que pour l'examen des erreurs d'inférence (III, 7).

Voici, en notant dans chaque cellule les paragraphes des éditions d'Abdelhamid I. Sabra et de A. Mark Smith, le plan du Livre III (Tableau 1) :

\footnotetext{
${ }^{35}$ «Quand la vue perçoit des objets dont les distances ne peuvent pas être certifiées, la faculté du jugement conjecture immédiatement leurs grandeurs [des distances] en comparant leurs distances avec celles des objets que la vue a déjà perçus et dont elle a certifiée les grandeurs [des distances] », Ibid., II, 3.87 ; Sabra, Optics, I, p. 156 ; Smith, Alhacen's, I, p. 135-136.
} 


\begin{tabular}{|c|c|c|c|c|c|c|c|c|c|c|}
\hline \multicolumn{2}{|c|}{ Sensibles (II, 3) $\nabla$} & Distance & Position & Lumière & Taille & Opacité & Transp. & Durée & Vision & \multirow{3}{*}{$\begin{array}{l}\text { (III, 5) } \\
(\mathrm{III}, 6)\end{array}$} \\
\hline Lumière & \multirow{2}{*}{$\S 49-66$} & $\S 4-6$ & 7 & 8 & 9 & 10 & 11 & $12-13$ & $14-15$ & \\
\hline Couleur & & $\S 4-7$ & $8-10$ & $11-13$ & $14-15$ & $16-17$ & $18-24$ & $25-26$ & $27-31$ & \\
\hline Distance & $67-93$ & $\S 2-3$ & $63-64$ & $104-105$ & $131-133$ & \multirow{2}{*}{163} & 193-194 & 217 & $250-253$ & \multirow[t]{20}{*}{ (III, 7) } \\
\hline Position & $94-120$ & $4-7$ & $65-66$ & 106 & $134-136$ & & 195 & $218-220$ & $254-255$ & \\
\hline Volume & $121-126$ & $8-10$ & $67-70$ & 107 & 137 & 164 & 196 & 221 & 256 & \\
\hline Forme & $127-134$ & $11-12$ & $0 \%-10$ & 107 & 131 & 104 & 150 & 221 & 250 & \\
\hline Taille & $135-171$ & $13-25$ & $71-74$ & $110-111$ & $140-141$ & 165 & 197 & $222-225$ & - & \\
\hline Séparation & $172-174$ & $26-28$ & $75-76$ & \multirow{3}{*}{$112-115$} & \multirow{3}{*}{$142-144$} & \multirow{3}{*}{$166-169$} & \multirow{3}{*}{$198-201$} & \multirow{3}{*}{$226-229$} & \multirow{2}{*}{257} & \\
\hline Continuité & $175-176$ & $29-30$ & $77-78$ & & & & & & & \\
\hline Nombre & 177 & 31 & $79-80$ & & & & & & $258-260$ & \\
\hline Mouvement & 178-187 & $32-33$ & $81-82$ & 116 & $145-146$ & \multirow{2}{*}{$170-172$} & $202-203$ & $230-231$ & $261-263$ & \\
\hline Repos & 188 & $34-38$ & $83-84$ & 117 & $147-148$ & & 204-205 & $232-236$ & $264-266$ & \\
\hline Texture & 189-191 & $39-43$ & $85-86$ & \multirow{4}{*}{$118-120$} & $149-150$ & $173-1$ & \multirow{6}{*}{$206-210$} & \multirow{2}{*}{$237-238$} & \multirow{4}{*}{$267-271$} & \\
\hline Planéité & 192-194 & $44-45$ & $87-88$ & & 149 & $1 / 5-$ & & & & \\
\hline Transp. & $195-196$ & $46-47$ & \multirow{2}{*}{$89-91$} & & $151-153$ & $175-177$ & & $239-240$ & & \\
\hline Opacité & 197 & $48-49$ & & & & & & & & \\
\hline Ombre & 198 & $50-51$ & $92-93$ & \multirow{2}{*}{$121-123$} & \multirow{2}{*}{$154-155$} & $178-179$ & & \multirow{2}{*}{$241-243$} & \multirow{2}{*}{$272-274$} & \\
\hline Obscurité & 199 & $52-53$ & $94-95$ & & & 180 & & & & \\
\hline Beauté & $200-231$ & $54-55$ & $96-97$ & \multirow{2}{*}{$124-126$} & \multirow{2}{*}{$156-158$} & \multirow{4}{*}{$185-192$} & \multirow{4}{*}{$211-216$} & \multirow{4}{*}{$244-249$} & \multirow{4}{*}{$275-278$} & \\
\hline Laideur & 232 & $56-57$ & 98-99 & & & & & & & \\
\hline Similarité & 233 & $58-59$ & $100-101$ & \multirow{2}{*}{$127-130$} & \multirow{2}{*}{$159-162$} & & & & & \\
\hline Différence & $234-235$ & $60-62$ & $102-103$ & & & & & & & \\
\hline
\end{tabular}

Tableau 1

Si l'on suit la logique combinatoire du Livre III, dont le plan a été soigneusement pensé par Ibn al-Haytham, il faut : $1^{\circ}$ déterminer comment l'extinction atmosphérique se rapporte aux erreurs de perception visuelle en général (perspective aérienne) ; $2^{\circ}$ déterminer comment cettethéorie des erreurs de perception rend compte du grandissement des astres à l'horizon (illusion lunaire). L'hypothèse que nous faisons ici est qu'une analyse de ces questions doit s'émanciper d'une lecture classique des textes, fondée sur l'emboîtement des unités contextuelles (phrases, paragraphes, chapitres), pour adopter une lecture « verticale » référantsans cesse les plus petites unités au plan d'ensemble qui les organise.

Au chapitre III, 7, Ibn al-Haytham étudie les erreurs causées par un défaut de transparence (shafíf/diaphanitas) du milieu. Cette analyse est irréductible à la théorie physique exposée au Livre VII, où elle n'est d'ailleurs qu'à peine évoquée : $1^{\circ} \mathrm{Ibn}$ al-Haytham disant que la vue $s e$ trompe, il présente une théorie où des facteurs psychologiques (erreurs d'inférence) collaborent avec un facteur physique (extinction atmosphérique); $2^{\circ}$ Il parle toujours en association $d u$ brouillard et de la poussière, laquelle est composée de particules matérielles qui ne produisent aucune réfraction ; $3^{\circ}$ Lorsqu'il compare le grandissement de laLune et celui d'un objet plongé dans l'eau, il ne vise pas à transposer la théorie de la réfraction aux vapeurs de l'atmosphère. Le rapprochement est purement factuel : la Lune grandit à l'horizon comme la pierre grandit dans l'eau, état de fait qui n'implique en rien la réfraction. Mais quelle autre cause mobiliser, sinon l'extinction qui se produit aussi bien dans l'eau que dans l'air? 
Selon Ibn al-Haytham, l'extinction atmosphérique peut causer une erreur de jugement : $1^{\circ}$ quant à la taille directement (miqdâr/quantitas-mensura) ; $2^{\circ}$ quant à la distance (bu'd/remotiospatium) donc quant à la taille ; $3^{\circ}$ quant à la volumétrie(tajassum/corporeitas), donc quant à la distance et quant à la taille ; $4^{\circ}$ quant à la texture (khushûna/asperitas), donc quant à la distance et quant à la taille. Les passages-clefs sont les suivants :

\subsection{Extinction $\rightarrow$ Taille}

Extinction $\rightarrow$ Taille : «Maintenant si l'objet visible est dans le brouillard ou la poussière, et si l'oeil est situé dans l'air pur, la vue percevra la taille de l'objet plus grande qu'en réalité [...] Et quand la vue perçoit un objet plus grand qu'il n'est en réalité, alors elle se trompe quant à la taille de l'objet » ${ }^{36}$.

\subsection{Extinction $\rightarrow$ Distance $\rightarrow$ Taille}

Extinction $\rightarrow$ Distance : «La vue ne peut pas certifier la grandeur de la distance d'un objet vu à travers le brouillard ou la poussière [...] Mais si la vue perçoit un objet sans certifier la grandeur de la distance de l'objet $[\ldots]$ elle peut croire l'objet éloigné alors qu'il est proche en réalité $[\ldots]$ alors elle se trompe quant à la distance de l'objet ${ }^{37}$.

Distance $\rightarrow$ Taille $:$ «Lorsque la distance de l'objet est assurée, et qu'elle est modérée relativement à cet objet, la taille perçue de cet objet est certifiée ; si la distance de l'objet n'est pas connue, alors sa taille n'est pas certifiée $»^{38}$.

\subsection{Extinction $\rightarrow$ Volumétrie $\rightarrow$ Distance $\rightarrow$ Taille}

Extinction $\rightarrow$ Volumétrie : « Si la surface de l'objet présente une convexité ou une concavité, ou si ses parties ne sont pas semblablement situées, la vue ne percevra pas cette convexité ou concavité si la perception a lieu dans le brouillard ou la poussière. [...] Mais si la vue croit qu'une surface convexe ou concave est plane, alors elle se trompe quant à la forme de la surface $»^{39}$.

Volumétrie $\rightarrow$ Distance : «Une erreur peut aussi survenir dans l'appréciation de la courbure d'un corps situé à grande distance. Si la convexité ou la concavité d'un corps est tournée vers l'œil, un tel corps incurvé sera vu plat, parce que la courbure n'est rendue perceptible que par la différence entre les distances des parties du corps. En percevant que le centre de l'objet, ou un point voisin, est plus éloigné de l'œil que les bords, la vue percevra l'objet incurvé et convexe dans la direction de l'œil. Mais lorsque l'objet

\footnotetext{
${ }^{36}$ Ibid., III, 7.197 ; Sabra, Optics, I, p. 341 ; C'est moi qui souligne les mots brouillard, fumée et poussière, de même que dans les passages qui suivent ; la version latine est plus courte, Smith, Alhacen's, I, p. 330.

${ }^{37}$ Ibid., III, 7.193 ; Sabra, Optics, I, p. 340 ; Smith, Alhacen's, I, p. 329. Dans ce passage, Ibn al-Haytham étudie deux erreurs opposées : on peut percevoir un objet plus proche qu'il n'est en réalité, ou plus éloigné qu'il n'est en réalité. L'illusion lunaire est apparentée au second cas.

${ }^{38}$ Ibid., III, 7.17 ; Sabra, Optics, I, p. 286 ; la version latine ajoute ici que la grandeur de la distance est connue par le biais des objets interposés, Smith, Alhacen's, I, p. 306.

${ }^{39}$ Ibid., III, 7.196 ; Sabra, Optics, I, p. 341 ; la version latine est écourtée, Smith, Alhacen's, I, p. 330.
} 
est trop éloigné [...], la vue ne percevra pas la différence entre la distance de son milieu et celle de ses bords par rapport à l'oeil » ${ }^{40}$.

Distance $\rightarrow$ Taille : «Il a été montré, en outre, que lorsque la distance de l'objet est assurée, et quand elle fait partie des distances modérées relativement à cet objet, la taille perçue de cet objet est certifiée ; mais si la distance de l'objet n'est pas certaine, alors sa taille perçue ne l'est pas davantage $»^{41}$.

\subsection{Extinction $\rightarrow$ Texture $\rightarrow$ Position $\rightarrow$ Distance $\rightarrow$ Taille}

Extinction $\rightarrow$ Texture $:$ «Une erreur quant à texture [...] peut se produire à cause de la densité de l'air. Car, en regardant une surface finement texturée dans le brouillard, la fumée ou une épaisse poussière, la vue ne parviendra pas à percevoir sa rugosité car elle ne peut pas certifier la forme d'une surface vue dans le brouillard, la fumée ou une épaisse poussière $»^{42}$.

Texture $\rightarrow$ Position : «La rugosité d'un corps finement texturé ne sera pas visible à grande distance, même si celle-ci n'est pas excessive. Car la vue perçoit la texture seulement en percevant les différentes positions des parties de la surface du corps. [...] Quand la texture est fine, les différences de position des parties de l'objet seront petites et, en conséquence, la vue ne sera pas capable de les discerner à grande distance, même si celle-ci n'est pas excessive. [...] Par conséquent, lorsque la vue perçoit un corps texturé situé à de très grandes distances, elle échouera à percevoir sa texture $»^{43}$.

Position $\rightarrow$ Distance : « [La suite concerne] la façon dont la vue se trompe en inférant la distance lorsque la position de l'objet sort d'un registre modéré. [...] La vue percevra ces deux objets [i.e. séparés] comme contigus ou proches l'un de l'autre, et ne ressentira pas la distance entre eux, si l'observateur ne les connait pas antérieurement et s'il n'a vu pas précédement la distance qui les sépare. [...] Mais si la vue perçoit deux objets séparés l'un de l'autre, comme contigus ou proches l'un de l'autre, alors elle se trompe quant à la distance de l'objet le plus éloigné $»^{44}$.

Distance $\rightarrow$ Taille $:$ «Lorsque la distance de l'objet est assurée, et quand elle fait partie des distances modérées relativement à cet objet, la taille perçue de cet objet est certifiée ; mais si la distance de l'objet n'est pas certaine, alors sa taille perçue ne l'est pas davantage $»^{45}$.

Cette contribution de l'extinction aux erreurs d'inférence constitue une théorie infiniment plus synthétique que les causes accidentelles et permanentes exposées au Livre VII, parce qu'elle fait fond sur l'ensemble de la théorie de la perception proposée par Ibn al-Haytham.

\footnotetext{
${ }^{40}$ Ibid., III, 7.10 ; Sabra, Optics, I, p. 282 ; la version latine ramène l'examen de la convexité et de la concavité des corps au cas unique de la courbure, Smith, Alhacen's, I, p. 304.

${ }^{41}$ Ibid., III, 7.17 ; Sabra, Optics, I, p. 286 ; Smith, Alhacen's, I, p. 306.

${ }^{42}$ Ibid., III, 7.206 ; Sabra, Optics, I, p. 344 ; la version latine fusionne ici l'examen du rugueux et du lisse, Smith, Alhacen's, I, p. 330.

${ }^{43}$ Ibid., III, 7.44 ; Sabra, Optics, I, p. 297 ; Smith, Alhacen's, I, p. 312.

${ }^{44}$ Ibid., III, 7.63 ; Sabra, Optics, I, p. 303 ; la version latine est écourtée, Smith, Alhacen's, I, p. 314.

${ }^{45}$ Ibid., III, 7.17 ; Sabra, Optics, I, p. 286 ; Smith, Alhacen's, I, p. 306.
} 


\section{La théorie de l'illusion lunaire dérivée du Livre III}

Étudions maintenant comment Ibn al-Haytham a appliqué la théorie de la perspective aérienne du Livre III de l'Optique pour rendre compte de l'illusion lunaire.

\subsection{Note sur l'extinction et la perte de luminance}

Dans ses réponses à Ibn Ma‘dân, Ibn al-Haytham mentionne tout d'abord l'extinction atmosphérique sans en faire la base d'une théorie de l'illusion lunaire :

«Quand le Soleil est sur l'horizon, la vue est capable de le regarder bien en face et de continuer de le contempler sans éprouver de douleur ou d'infirmité comme résultat. Quand le Soleil monte au-dessus de l'horizon ou occupe le milieu du ciel, la vue cesse d'être capable de le regarder ou de le contempler $»^{46}$.

Quand l'astre est haut dans le ciel, il est lumineux et sa vue est douloureuse. Quand il est proche de l'horizon, nous le voyons sans douleur, les vapeurs et les poussières sur l'horizon lui faisant perdre sa luminosité. Ce passage ne fait pas explicitement référence au problème des erreurs d'inférence de la taille. Cet effet de l'extinction de la lumière sur la taille perçue des corps est décrit au livre III : «Si l'objet visible est dans le brouillard ou la poussière, et si l'oeil est situé dans l'air pur, la vue percevra la taille de l'objet plus grande qu'en réalité, de la même façon qu'elle perçoit des objets dans l'eau ${ }^{47}$. Son application au problème de l'illusion lunaire apparaît dans les réponses d'Ibn al-Haytham à Ibn Ma‘dân, un traité postérieur à l'Optique :

«Quant au grandissement [des astres] à l'horizon, nous ne le percevons pas à cause de leur proximité et de leur distance réduite par rapport à nous, mais parce qu'une vapeur humide qui entoure la terre s'interpose entre notre œil et eux, et c'est pourquoi ils sont vus ainsi, exactement comme ce qui est jeté dans l'eau est vu plus grand, et qu'il paraît plus grand à mesure qu'il s'enfonce $»^{48}$.

Ibn al-Haytham précise bien : "Ptolémée ne compare pas le grandissement des astres à l'horizon aux objets jetés dans l'eau à cause de ce qu'il a montré dans l'Optique [i.e. la réfraction], mais assimile plutôt [les astres] à ce qui est jeté dans l'eau parce que les choses sont ainsi $»^{49}$. Ce passage, comme celui dans lequel il est dit que « la vue perçoit un objet plus grand, de

\footnotetext{
${ }^{46}$ Solution des doutes sur l'Almageste, II, 3, Sabra, « On seeing the stars, II », p. 45.

${ }^{47}$ Kitâb al-manâzir , III, 7.197 ; Sabra, Optics, I, p. 341 ; Smith, Alhacen's, I, p. 330.

${ }^{48}$ Sabra, «On seeing the stars, II », p. 32.

${ }^{49}$ Sabra, «On seeing the stars, II », pp. 38, 54-55. Ptolémée écrit : «Car si les astres nous paraissent plus grands quand ils sont dans l'horizon [...] c'est à cause de la vapeur humide qui environne la terre entre nos yeux et les astres, comme les choses plongées dans l'eau nous y paraissent plus grandes qu'elles y sont plus profondément enfoncées », KАAY
} 
la même façon qu'elle perçoit un objet dans l'eau », n'est pas à mettre au compte de la réfraction mais de l'extinction de la lumière. Par conséquent, lorsque les vapeurs et les poussières nous font voir le soleil sans douleur à l'horizon, notre habitude est trompée, nous le croyons plus éloigné ; mais comme son diamètre angulaire est identique, nous le croyons plus grand.

\title{
7.2. Note sur la distance et les objets interposés
}

Le texte d'Ibn al-Haytham est le suivant :

\begin{abstract}
« Nous disons que la vue perçoit tout astre au zénith plus petit qu'en n'importe quelle autre région du ciel dans laquelle l'astre voyage $[\ldots]$ et que l'astre paraît plus grand à l'horizon. [...] Nous avons montré que la vue ne perçoit et ne certifie la grandeur des distances des objets que si ces distances sont jalonnées d'objets contigus et rapprochés. [...] Mais les distances des astres ne sont pas jalonnées d'objets contigus. Par conséquent, la vue ne peut pas percevoir et certifier leurs grandeurs, mais seulement les conjecturer en assimilant la distance des astres à celle des objets terrestres très éloignés qu'elle peut percevoir et dont elle conjecture la grandeur $»^{50}$.
\end{abstract}

L'extinction atmosphérique provoque une erreur d'appréciation de la distance et, par suite, de la taille. Ibn al-Haytham donne l'explication suivante de l'illusion lunaire : l'inférence correcte d'une distance suppose que celle-ci soit jalonnée d'objets contigus et rapprochés, ce qui n'est pas le cas des objets célestes. La vue fait alors une conjecture en assimilant la distance de la Lune à la distance d'objets terrestres éloignés ${ }^{51}$. Lorsque la vue est troublée par la présence de vapeurs ou de poussière, les objets paraissent plus éloignés mais, conservant le même diamètre angulaire, ils sont jugés plus grands. Ibn al-Haytham soutient ici la théorie de l'inférence luminance-distance, typique de la tradition ptoléméenne.

\subsection{Note sur la forme des corps convexes}

Au chapitre III, 7 de l'Optique, Ibn al-Haytham soutient que l'extinction peut causer une erreur d'appréciation de la volumétrie. Il s'ensuivrait une erreur de jugement de distance, donc de taille. L'idée est reprise au Livre VII, sous la forme d'une observation qui introduit sa théorie psychologique du ciel aplati, mais qui vaut comme explication autonome de l'illusion lunaire.

que de Claude Ptolémée, trad. M. Halma (Paris, 1813), t. I, p. 9. L’analogie est fréquente chez Ptolémée, cf. Optica, II, 9, A. Lejeune, L'Optique de Claude Ptolémée, p. 14.

${ }^{50}$ Kitâb al-manâzirir, VII, 7.55 ; Sabra, «Psychology versus mathematics », pp. 237-238 ; Optica thesaurus, éd. Risner, p. 280. L'édition de Risner n'étant pas une édition critique, il faudra attendre la publication par Smith des livres VI et VII du De Aspectibus pour apprécier les différences entre les versions arabes et latines.

${ }^{51}$ «Quant à la raison pour laquelle ce qui apparaît à la vue et est imaginé relativement à la taille des planètes n'est pas en rapport avec leurs distances, nous devons reconnaître que c'est là une erreur qui affecte la vision à cause de la différence des perspectives. [...] Tout astre est vu plus près de nous qu'il ne l'est en réalité, parce que 
«Et quand la vue ne peut identifier une chose, elle l'assimile à un objet familier qui lui ressemble. Ainsi, elle perçoit le Soleil et la Lune comme étant plats, et perçoit les corps concaves et convexes, situés à de très grandes distances, comme étant plats. Elle perçoit aussi les arcs dont la convexité ou la concavité est tournée vers l'œil, comme droits. Car, lorsque la vue ne distingue pas [dans le cas des corps convexes] la proximité de leur centre et l'éloignement de leurs extrémités, [...] elle assimile les surfaces convexes à des surfaces planes, et assimile les arcs à des lignes droites, parce que les objets les plus familiers ont des surface planes et des arêtes droites $»^{52}$.

Si la vue ne peut pas distinguer le premier plan et l'arrière plan d'un corps convexe, elle assimile ce corps à une surface plane. Mais les deux objets présentant le même diamètre angulaire et l'objet plan étant plus éloigné que le convexe, cet éloignement revient à surestimer la taille de l'objet plan. Cette confusion s'applique au corps sphérique de la Lune : l'extinction atmosphérique neutralise le contraste de luminance et le relief. Sa distance et sa taille sont alors naturellement surestimées.

\subsection{Note sur la texture des corps}

Une autre explication des erreurs de vision est fondée sur la cascade causale selon laquelle un défaut de transparence du milieu cause une perte de texture du corps, qui provoque à son tour une mauvaise appréciation de sa distance et de sa taille. L'habitude que nous avons d'un corps visible fait que nous pouvons inférer sa distance à partir des détails qui sont perceptibles à sa surface : plus sa distance est grande, moins nous en percevons les détails. Lorsque l'air devient plus dense, les détails visibles à la surface du corps disparaissent, ce qui nous pousse à surévaluer sa distance. Mais le diamètre angulaire du corps étant constant, sa taille paraît alors plus grande. Ibn al-Haytham disposait de tous les éléments pour interpréter l'illusion lunaire à partir de cette relation qui apparaît au Livre III, cf. supra 6.4. Or ce passage-clef n'a pas été appliqué à la perception de la taille des astres. Pourquoi ? Cela paraît découler des thèses qu'Ibn alHaytham a soutenu à propos de la lumière de la Lune. Dans un premier traité, intitulé Sur la lumière de la Lune, Ibn al-Haytham conçoit parfaitement : $1^{\circ}$ que la Lune est un corps sphérique ; $2^{\circ}$ qu'elle n'a pas de lumière propre ; $3^{\circ}$ que sa lumière n'est pas celle d'une réflexion sur un corps poli. Mais il ne trouve pas la voie qui l'aurait conduit au concept de réflexion diffuse :

la vue se fonde sur les distances auxquelles elle est habituée et qu'elle juge familières », Ptolémée, Hypothèses planétaires ; Sabra, « Psychology versus mathematics », pp. 221-222.

${ }^{52}$ Kitâb al-manâżir, VII, 7.55 ; Sabra, "Psychology versus mathematics», p. 238 ; Optica thesaurus, éd. Risner, p. 281. Nous lisons ce passage indépendament du terme de la démonstration, qui est d'établir que la concavité du ciel n'est pas perçue, puisque tous les corps concaves ou convexes situés à de très grandes distances paraissent aplatis, cf. supra 4.1. La volumétrie est un facteur négligeable par rapport aux précédents. 
«Le fait que l'on constate exactement la même chose, quel que soit l'endroit ou le moment où l'on fait l'expérience, montre que la lumière émane de toute la surface lumineuse de la Lune vers tout point se trouvant vis-à-vis d'elle [...] Nous abordons maintenant notre propre réponse [...] Le corps de la Lune, lorsqu'il est éclairé par le Soleil, devient lumineux en soi, la lumière qui en émane et qui s'étale sur la terre devient alors la lumière de son propre corps $[\ldots]$ comme si le Soleil, en rayonnant sur la Lune, lui accordait une certaine forme, grâce à laquelle sa substance devient lumineuse, et que, par conséquent, sa lumière émane de la même façon que la lumière émane à partir des corps lumineux en soi $\aleph^{53}$.

Dans le second traité, Sur la nature de la tache qui apparaît sur la face de la Lune, Ibn alHaytham écarte à nouveau l'idée que la lumière de la Lune puisse résulter d'une réflexion :

\footnotetext{
«Quelqu'un pourrait dire cependant que ce dont témoigne la réalité, c'est que la lumière apparaissant sur les surfaces des corps polis, au moment où la lumière y rayonne, est une lumière fort éclatante, plus forte que celle qui apparaît sur les surfaces des corps rugueux, et que cela peut servir de preuve que les corps polis reçoivent plus de lumière que les corps rugueux [...] Donc la puissance de la lumière perçue par l'œil sur la surface de la Lune n'est pas due à son poli, et la faiblesse de la lumière perçue à l'endroit où il y a la tache n'est pas due à ses aspérités $»^{54}$.
}

Ces deux développements interdisaient à Ibn al-Haytham de concevoir le concept de réflexion diffuse et, par suite, d'envisager l'illusion lunaire comme une erreur d'inférence de taille fondée sur une mauvaise appréciation de la texture du corps, thème qui est pourtant développé au livre III.

\section{Conclusion}

Ibn al-Haytham a-t-il substitué une interprétation psychologique de l'illusion lunaire à une première théorie optico-géométrique, ou a-t-il présenté plusieurs causes articulées les unes aux autres, toutes dérivées de sa théorie des erreurs d'inférence (Kitâb al-manâzirir, III, 7) ? Les arguments qui plaident en faveur de la deuxième solution sont les suivants : $1^{\circ}$ dans la tradition ptoléméenne, développée sur ce point par Ibn al-Haytham, le rôle des vapeurs est irréductible à la réfraction puisqu'elles sont indépendamment responsables de l'extinction atmosphérique ; $2^{\circ}$ la double explication par les causes permanente et accidentelle est exposée seulement au Livre VII, intégralement dédié à l'étude de la réfraction. Pour la discussion des erreurs d'inférence de taille - dont dépend l'illusion lunaire - ce livre est marginal par rapport au Livre III, qui contient la théorie physico-psychologique que nous venons d'exposer.

\footnotetext{
${ }^{53} \mathrm{H}$. Masoumi Hamadani, L'Optique et la physique céleste. L'œuvre optico-cosmologique d'Ibn al-Haytham, thèse de doctorat Paris VII (Paris, 2006), I, pp. 25-27.

${ }^{54}$ Ibid., pp. 101-102.
} 
Cette explication peut être résumée ainsi : 1. L'illusion lunaire est un problème de perception de la taille. 2. La taille est perçue par la comparaison du diamètre angulaire et de la distance. 3. Le diamètre angulaire étant constant, l'illusion résulte d'une erreur de jugement quant à la distance, qui ne peut être ici que conjecturée. 4. L'extinction atmosphérique affecte : [1] le jugement de taille directement ; [2] l'estimation de la distance, donc de laaille ; [3] le jugement relatif à la volumétrie, donc à la distance et à la taille. Quant à la perception de la texture, et ses conséquences sur l'inférence de taille, elle n'a pas été appliquée par Ibn al-Haytham à l'illusion lunaire. C'est dommage, car nous aurions alors disposé d'une interprétation de l'illusion lunaire faisant d'Ibn al-Haytham le précurseur de l'approche écologique défendue par Gibson, selon laquelle la perception de la texture caractéristique d'une surface varie en fonction de la distance. Si la « quantité de texture »(amount of texture) diminue, alors l'objet est perçu plus éloigné. Par suite, il paraît plus grand qu'il n'est en réalité ${ }^{55}$.

La théorie de l'extinction atmosphérique proposée par Ibn al-Haytham donne tout son sens au concept de perspective aérienne de Léonard de Vinci. Son caractère intégrateur (collaboration de facteurs physiques et psychologiques) facilitait son acclimatation en peinture : une théorie purement psychologique n'aurait pas suggéré de possibilité d'action sur le réel ; une théorie purement physique n'aurait signalé ni la difficulté, ni l'intérêt, d'une restitution picturale. Le caractère général de cette théorie - l'enchaînement extinction-texture-volumétrie-distance-taille n'étant pas référé au seul cas de l'illusion lunaire - facilitait son émancipation et son application à des sujets variés. On comprend alors comment Vinci, mis au contact de l'optique d'Ibn alHaytham et de ses commentateurs latins, a pu tirer le concept de «perspective aérienne »de cette explication des erreurs de perception visuelle fondée sur l'extinction atmosphérique.

Je tiens à remercier les deux lecteurs anonymes qui m'ont aidé, par leurs observations, à clarifier plusieurs points qui étaient restés implicites dans une première version de cet article.

${ }^{55}$ J. J. Gibson, The Ecological Approach to Visual Perception (London, 1986), pp. 23-24, 162. 